INPLASY

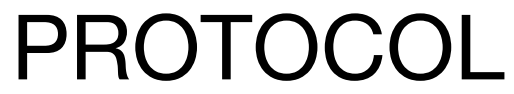

To cite: Chen et al. Effects of acupuncture on lung function in adults patients with asthma: a protocol for systematic review and meta-analysis. Inplasy protocol 202160022. doi:

10.37766/inplasy2021.6.0022

Received: 07 June 2021

Published: 07 June 2021

Corresponding author: Yaxuan Chen

511677913@qq.com

Author Affiliation:

Department of

Anesthesiology, The First

Affiliated Hospital of

Guangzhou University of

Chinese Medicine,

Guangzhou, China.

Support: None.

Review Stage at time of this submission: The review has not yet started.

Conflicts of interest:

None declared.

\section{Effects of acupuncture on lung function in adults patients with asthma: a protocol for systematic review and meta-analysis}

Chen, Y1; Yang, C2; Zheng, J3; Ma, W4.

Review question / Objective: The purpose of this systematic review was to evaluate the effectiveness of acupuncture therapy of asthma and to demonstrate which type of acupuncture is more effective.

Condition being studied: Adults patients with asthma.

Information sources: The searched database include Pubmed, Web of Scince, Embase, Cochrane, China National Knowledge Infrastructure(CNKI), Chinese Biomedical Literature Databases(CBM), Wanfang database and Viper database.

INPLASY registration number: This protocol was registered with the International Platform of Registered Systematic Review and Meta-Analysis Protocols (INPLASY) on 07 June 2021 and was last updated on 07 June 2021 (registration number INPLASY202160022).

\section{INTRODUCTION}

Review question / Objective: The purpose of this systemetic review was to evaluate the effectiveness of acupuncture therapy of asthma and to demonstrate which type of acupuncture is more effective.

Condition being studied: Adults patients with asthma. 


\section{METHODS}

Participant or population: Patients aged $\geq$ 18 years old who were diagnosed as asthma.

Intervention: The interventions in experimental group can be any type of acupuncture, without limitation of needle meterial, duration of treatment and operation methods.

Comparator: The control group received internationally recongnized therapy, such as glucocorticoid, or sham acupuncture treatment.

Study designs to be included: Randomized controlled trials.

Eligibility criteria: Observational studies, duplicate reports, animal studies, and studies without well described randomization methods will be excluded.

Information sources: The searched database include Pubmed, Web of Scince, Embase, Cochrane, China National Knowledge Infrastructure(CNKI), Chinese Biomedical Literature Databases(CBM), Wanfang database and Viper database.

Main outcome(s): Peak expiratory flow (PEF), the forced expiratory volume in the first second (FEV 1) and the forced vital capacity (FVC).

Quality assessment / Risk of bias analysis: The risk of bias of all the included literatures will be assessed according to the Cochrane handbook for systematic review of intervention.

Strategy of data synthesis: We will use the software Revman 5.4 to conduct a meta analysis.

Subgroup analysis: If high heterogeneity is found after data synthesis, subgroup analysis will be conducted according to different types of acupuncture methods, different acupuncture points and different lung function detection indexes.
Sensitivity analysis: We will conduct sensitivity analysis by eliminating studies one by one and repeating meta-analysis.

Country(ies) involved: China.

Keywords: asthma; acupuncture; meta analysis.

Contributions of each author:

Author 1 - Yaxuan Chen.

Email: 511677913@qq.com

Author 2 - Caiqi Yang.

Email: 1329103217@qq.com

Author 3 - Junyi Zheng.

Email: dswzheng@163.com

Author 4 - Wuhua Ma.

Email: gzmwh@aliyun.com 